\title{
Increased ERCC Expression Correlates with Improved Outcome of Patients Treated with Cisplatin as an Adjuvant Therapy for Curatively Resected Gastric Cancer
}

\author{
Sun Kyung Baek, M.D. ${ }^{1}$, Si-Young Kim, M.D. ${ }^{1}$, Jae Jin Lee, M.D. ${ }^{1}$, Yoon Wha Kim, M.D. ${ }^{2}$, Hwi Joong Yoon, \\ M.D. ${ }^{1}$ and Kyung Sam Cho, M.D. ${ }^{1}$
}

Departments of ${ }^{1}$ Internal Medicine, ${ }^{2}$ Anatomical Pathology, Cancer Research Institute, Kyung Hee University College of Medicine, Seoul, Korea

Purpose: It has been reported that the overexpression of the excision repair cross-complementing 1 (ERCC1) gene, which is essential for the repair of cisplatin (CDDP)DNA adducts, negatively influences the effectiveness of CDDP-based therapy for primary gastric cancer. We investigated whether the ERCC1 expression was associated with survival for gastric cancer patients in an adjuvant setting.

Materials and Methods: We retrospectively analyzed 44 patients who were diagnosed with stage II or higher disease after undergoing curative resection and they had also received cisplatin-based chemotherapy. The ERCC1 expression was examined by performing immunohistochemical (IHC) staining, and this was divided into two groups according to the percentage of IHC staining of the tumor cell nuclei (negative: $10 \%$ or less, positive: more than $10 \%$ ).

Results: Among the 44 patients (ERCC1-negativel

\section{INTRODUCTION}

Gastric cancer is ranked as the number one cause of cancer among Koreans (1), and this is a matter of serious concern among health care professionals. $30 \%$ to $40 \%$ of the patients who undergo curative resection for gastric cancer experience a recurrence of tumor, and the overall 5-year survival rate ranges from $10 \%$ to $40 \%$ (2). Despite the poor prognosis, gastric cancer has shown a considerable response to chemotherapy. Therefore, improving the survival of gastric cancer patients is now being done by modifying and developing the chemotherapeutic approach.

Cisplatin was discovered serendipitously by Rosenberg and

Correspondence: Si-Young Kim, Department of Internal Medicine, Kyung Hee University Hospital, 1 Hoegi-dong, Dongdaemun-gu, Seoul 130-701, Korea. (Tel) 82-2-958-8204, (Fax) 82-2-9681848, (E-mail) sykim55@chol.com

Received December 13, 2005, Accepted February 23, 2006
ERCC1-positive group=16/28), 32 patients were male and their median age was 52 years. There was no difference for the baseline characteristics of the two groups. The median follow-up duration was 41 months. The median disease-free survival (DFS) and the overall survival (OS) for the ERCC1-positive group were significant higher than those of the ERCC1-negative group (DFS: 40.4 vs. 14.6 months, $p=0.02$, OS: undefined vs. 20.4 months, $p=0.008$ ).

Conclusion: The overall survival in gastric cancer patients who received cisplatin-based adjuvant chemotherapy after a curative resection is higher in those patients showing the overexpression of the ERCC1 gene. However, prospective studies using the ERCC1 gene expression as a prognostic marker for the DNA repair activity are needed. (Cancer Res Treat. 2006;38:19-24)

Key Words: Adjuvant therapy, Cisplatin, ERCC1, Stomach neoplasms, Survival

colleagues (3) in 1965 as an inhibitor of bacteria growth, and it has a striking antitumor activity. By the mid 1970s, cisplatin was being administered to the patients suffering with germ cell tumors and this drug induced durable remissions and sometimes cures in many individuals suffering with metastatic disease (4). Cisplatin has now been proven effective against such common cancers such as those of the lung, the upper digestive tract, the urothelium and the ovarian epithelium.

The role of cisplatin for gastric cancer was first studied on patients with advanced disease. The previous phase II study showed a response rate from $23 \%$ to $73 \%$ for advanced gastric cancer treated with cisplatin-based combination therapy (5). Based on its effects on advanced gastric cancer, cisplatin has and is now being used as a basic agent in the adjuvant setting of gastric cancer. However, the antitumor activity of cisplatin has only been incompletely defined as an adjuvant therapy $(6,7)$, and its effects haven't be observed in all cases of advanced gastric cancer. The limited role of cisplatin for gastric cancer has been thought to be due to the cancer's mechanism of resistance, i.e., the expression of the excision repair crosscomplementing 1 (ERCC1) gene.

The ERCC1 gene prevents mutations and other injuries to 
the DNA via the nucleotide excision and repair pathway, and this pathway is essential for the repair of the cisplatin (CDDP)DNA adducts (9). Therefore, this gene may have a pivotal role in cancer cells' mechanism of resistance to cisplatin. It has been reported that the overexpression of the ERCC1 gene negatively influenced the effectiveness of CDDP-based therapy for advanced gastric cancer, non-small cell lung cancer, esophageal cancer and ovarian cancer $(10 \sim 14)$. However, there has been no study reporting on the expression of ERCC1 in the adjuvant setting of gastric cancer. This study investigated if the overexpression of the ERCC1 gene has an influence on the survival of the gastric cancer patients who received cisplatin-based adjuvant therapy after curative resection.

\section{MATERIALS AND METHODS}

\section{1) Patients}

From September 1996 to August 2004, 44 patients who were diagnosed with stage 2 or higher gastric cancer after a curative resection were retrospectively analyzed. All of the patients were aged 18 years or older, and they had received at least four cycles of cisplatin-based adjuvant chemotherapy. No other chemotherapy was given prior the adjuvant chemotherapy. The exclusion criteria included a prior history of malignancies, with the exception of basal cell carcinoma of the skin or carcinoma in situ of the cervix, or the presence of any active infection or other serious underlying medical conditions. The patients' characteristics were obtained by reviewing the patients' chart records. The patients were regularly followed up after they received the adjuvant chemotherapy.

\section{2) Administration of adjuvant cisplatin-based combi- nation chemotherapy}

After curative resection, if the patients had stage II or higher disease, according to TNM staging system (15), a good performance status and adequate laboratory findings, then we administered adjuvant cisplatin-based combination chemotherapy. Three cisplatin-based combination chemotherapeutic regimens were administered for the adjuvant chemotherapeutic regimens: the FP (5-fluorouracil and cisplatin), oFLP (oral 5-fluorouracil, leucovorin, cisplatin) or PELF (cisplatin, epirubicin, leucovorin and 5-fluorouracil) regimens.

The FP regimen was as follows. Cisplatin $100 \mathrm{mg} / \mathrm{m}^{2}$ was administered intravenously on day 1 and 5 -FU $1,000 \mathrm{mg} / \mathrm{m}^{2}$ was administered intravenously on days 1 to 5 . This regimen was repeated every three weeks. The oFLP regimen was cisplatin $100 \mathrm{mg} / \mathrm{m}^{2}$ administrated intravenously on day 1 , and oral 5-FU 500 $700 \mathrm{mg}$ and leucovorin $45 \mathrm{mg}$ were administrated orally on days 1 to 21 . This regimen was repeated every four weeks. Oral 5-FU (UFT) was administrated according to the body surface area (BSA): $1 \sim 1.5 \mathrm{~m}^{2}: 500 \mathrm{mg}, 1.5 \sim 2 \mathrm{~m}^{2}$ : $600 \mathrm{mg}$, and $>2 \mathrm{~m}^{2}: 700 \mathrm{mg}$. The PELF regimen consisted of two cycles of the PELF regimen and then this was followed by four cycles of the ELF regimen. The PELF regimen was as follows. Cisplatin $40 \mathrm{mg} / \mathrm{m}^{2}$ and epirubicin $30 \mathrm{mg} / \mathrm{m}^{2}$ were administered intravenously on days 1 and 5, and 5-FU 300 $\mathrm{mg} / \mathrm{m}^{2}$ and leucovorin $20 \mathrm{mg} / \mathrm{m}^{2}$ were administered intravenously on days 1 to 3 . This regimen was repeated every three weeks. The ELF regimen was epirubicin $75 \mathrm{mg} / \mathrm{m}^{2}$ administered intravenously on day 1 with the 5 -FU $450 \mathrm{mg} / \mathrm{m}^{2}$ and leucovorin $20 \mathrm{mg} / \mathrm{m}^{2}$ being administered on days 1 to 3 . This regimen was also repeated every three weeks.

\section{3) Assessment and evaluation}

All the patients underwent a medical history taking and a physical examination. Before the adjuvant treatment, assessments were conducted that included a $\mathrm{CBC}$, renal and liver function tests, urinalysis, ECG, a performance status check, chest X-ray, radionuclide bone scan and abdominal CT.

The toxicities and the routine laboratory exams were evaluated before the course of treatment. Follow-up evaluations were done after the treatment; these included laboratory tests, chest $\mathrm{X}$-rays, abdominal ultrasound and stool occult blood, and these exams were repeated every 3 months for 2 years, and then every 6 months until 5 years after treatment. Gastroscopy was repeated every 2 years.

\section{4) Immunohistochemical staining for the ERCC1 ex- pression}

Immunohistochemical (IHC) staining was performed by using a streptoavidin-biotin immunoperoxidase method according to the supplier's protocol (DAKO, LSAB kit, Carpenteria, CA). In brief, the paraffin-embedded tissue sections were deparaffinized in xylene and rehydrated with a graded series of ethanol solutions. After quenching the endogenous peroxidase activity by immersing the sections in $0.3 \%$ hydrogen peroxide for 30 minutes and then in blocking the reagents for 30 minutes, primary monoclonal mouse anti-human ERCC-1 (Clone 8F1, NeoMarkers, Fremont, CA) was applied to the sections at a dilution of $1: 100$, and the sections were allowed to incubate in a moist chamber for 2 hours at room temperature. After washing out the excess complex, the location of the antibodies was visualized by incubating the section for 10 minutes in 3,3'-diaminobenzidine tetrahydrochloride (DAKO, Carpinteria, CA). The positive control we used for ERCC-1 was the nuclear staining of the normal gastric epithelium that was adjacent to the cancer in each section. For the negative control, normal serum was substituted for the primary antibody.

\section{5) Statistical analysis}

We analyzed the disease-free survival (DFS) and the overall survival (OS) of the two groups, which were divided according to their ERCC1 expression. The survival rates were estimated using the Kaplan-Meier method. As for the prognostic factors, univariate and multivariate analyses were used for the DFS; the OSs were estimated with using log-rank and Cox regression tests. The prognostic factors of gastric cancer were analyzed with regard to the performance status, gender, age, the tumor stages, the expression of ERCC1, the operative method and the chemotherapy regimens. The patients' characteristics and the side effects between the two groups were also analyzed via the $\kappa^{2}$-test and Fisher's exact test (SPSS software, version 11.0; SPSS Inc, Chicago, IL). 


\section{RESULTS}

\section{1) Patient characteristics}

The characteristics of the 44 eligible patients are listed in Table 1. Among a total of 44 patients, 32 patients were male and 12 patients were female. The median age was 52 years, and the patients' ages ranged from 35 to 70 years. Sixteen patients were in the ERCC1-negative group and twenty-eight patients were in the ERCC1-positive group. There was no difference in their baseline characteristics i.e. age, stage, the number of cycles of chemotherapy and the operative method between the two groups, except for the number of cases with lymph node metastasis (Table 1). The proportion of patients with stage II and/or IIIA disease in each group was $44 \%$. All the patients received at least 4 or more cycles of CDDP-based adjuvant chemotherapy (PELF: 15, oFPLP: 18, FP: 11). The median follow-up period was 41 months.

\section{2) ERCC1 expression level}

The expression of the ERCC1 gene, as determined by the IHC staining, was divided into two groups according to grading the proportion of nuclei that were stained in the tumor cells. The grading system was as follows: if immunoreactivity was noted in less than $10 \%$ of the tumor cells, then we defined this as negative; if the immunoreactivity was $10 \%$ or more of the tumor cells, then we defined this as positive (Fig. 1).

Table 1. Demographics and treatment characteristics of the gastric cancer patients according to the ERCC1 expression level

\begin{tabular}{|c|c|c|c|c|c|}
\hline & & Total patients $(n=44)$ & ERCC1 negative $(n=16)$ & ERCC1 positive $(n=28)$ & p-value \\
\hline \multirow[t]{2}{*}{ Gender } & Male & 32 & $13(81 \%)$ & $19(60 \%)$ & 0.48 \\
\hline & Female & 12 & $3(19 \%)$ & $9(40 \%)$ & \\
\hline \multirow[t]{2}{*}{ Age } & Median & 52 & 56 & 49 & 0.25 \\
\hline & Range & $35 \sim 70$ & $35 \sim 65$ & $35 \sim 70$ & \\
\hline \multirow{4}{*}{ Stage* } & II & 1 & $0(0 \%)$ & $1(4 \%)$ & 0.32 \\
\hline & IIIA & 18 & $7(44 \%)$ & $11(39 \%)$ & \\
\hline & IIIB & 14 & $3(19 \%)$ & $11(39 \%)$ & \\
\hline & IV & 11 & $6(37 \%)$ & $5(18 \%)$ & \\
\hline \multirow[t]{3}{*}{ Cycles } & Four & 5 & $1(6 \%)$ & $4(14 \%)$ & 0.25 \\
\hline & Five & 3 & $0(0 \%)$ & $3(11 \%)$ & \\
\hline & Six & 36 & $15(94 \%)$ & $21(75 \%)$ & \\
\hline \multirow[t]{2}{*}{ Surgery } & $\mathrm{STG}^{\dagger}$ & 29 & $8(50 \%)$ & $21(80 \%)$ & 0.09 \\
\hline & $\mathrm{TG}^{\ddagger}$ & 15 & $8(50 \%)$ & $7(20 \%)$ & \\
\hline
\end{tabular}

${ }^{*}$ No. of metastatic lymph nodes, ERCC-negative vs. ERCC-positive, 13 vs. 8 , respectively $\mathrm{p}=0.02,{ }^{\dagger}$ subtotal gastrectomy, ${ }^{\ddagger}$ total gastrectomy.
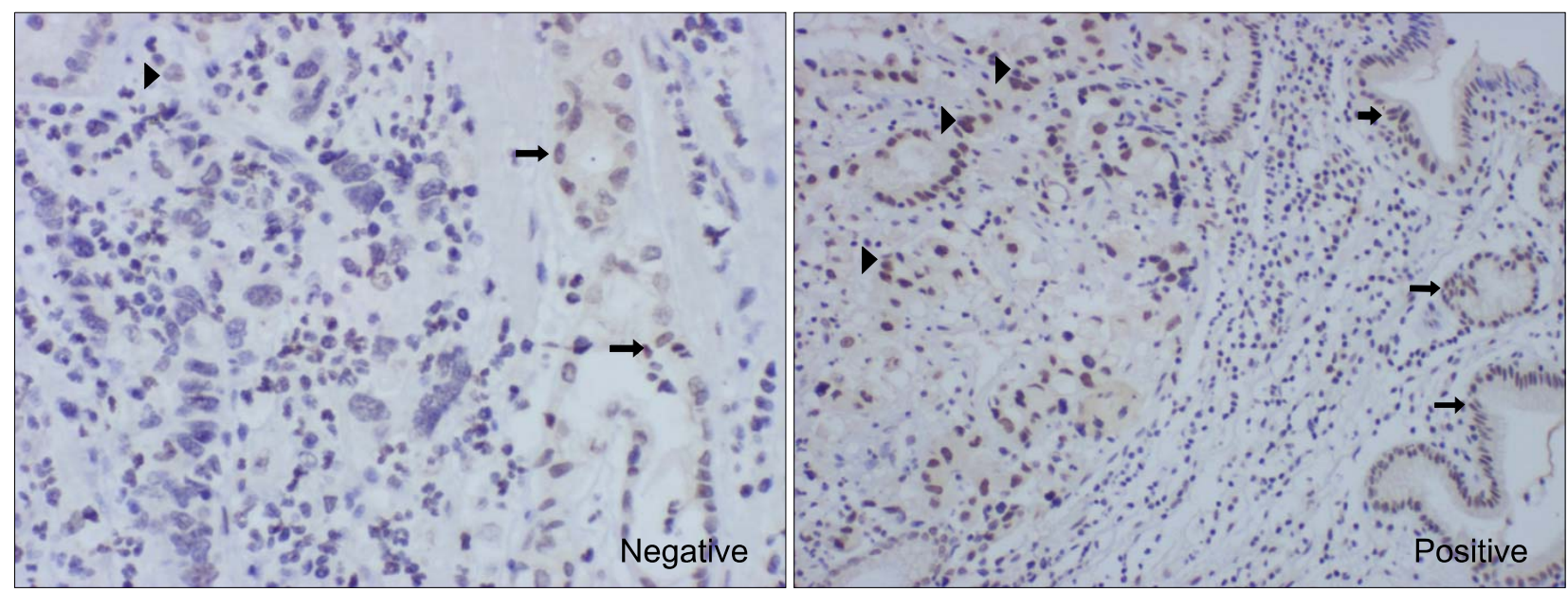

Fig. 1. The expression of ERCC1 in stomach cancer. Immunohistochemical staining for the ERCC1 gene in cancer cells is shown by brown nucleus staining; counterstaining was done with haematoxylin (arrow: stained normal cell, arrow head: stained cancer cell). Negative: $<10 \%$, Positive: more than $10 \%$. 


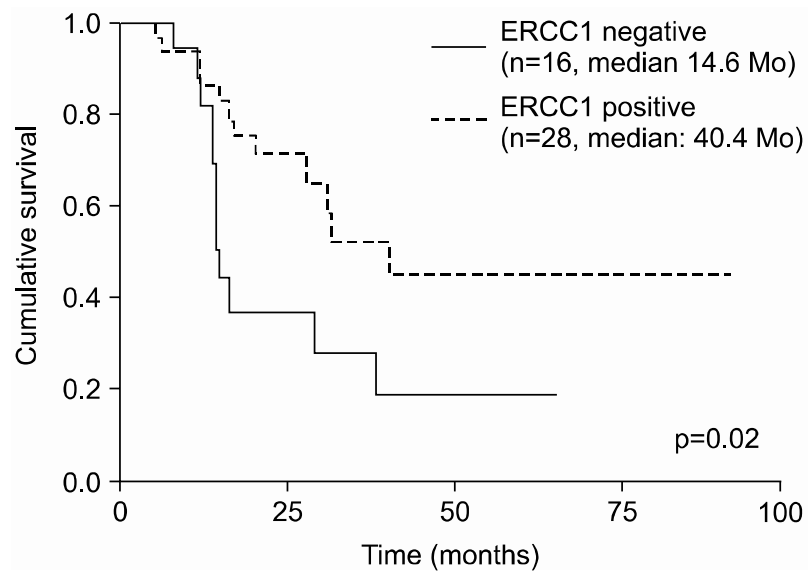

Fig. 2. Disease free survival according to more and less than a $10 \%$ ERCC1 expression level as the cutoff level.

\section{3) Disease-free survival and overall survival}

The median DFS was 40.4 months in the ERCC1-positive group and 14.6 months in the ERCC1-negative group ( $\mathrm{p}=0.02$ ) (Fig. 2). In terms of the median OS, the ERCC1-positive group had a significantly higher OS than did the ERCC1-negative group (undefined vs. 20.4 months, respectively, $\mathrm{p}=0.008$ ) (Fig. 3 ). Both the DFS and OS of the ERCC1-positive group were superior to those of the ERCC1-negative group.

\section{4) Relapse and second-line therapy}

Of the 44 patients, twenty-four patients $(55 \%)$ had tumor recurrence and seventeen patients (39\%) died. According to the ERCC1 expression, 12 patients (75\%) in the ERCC1-positive group and 12 patients $(43 \%)$ in the ERCC1-neagtive group relapsed. Although 8 of the 24 relapsed patients were treated with second-line therapy, only 5 patients received at least three cycles of second-line chemotherapy. Only one patient of 12 relapsed patients in the ERCC1-negative group was treated with oxaliplatin combination chemotherapy as the second-line therapy and the patient is still alive. 4 of the 12 relapsed patients in the ERCC1-positive group were treated with secondline chemotherapy and 2 of these patients are still alive.

\section{DISCUSSION}

The ERCC1 gene is operant in all mammalian cells; it encodes a protein made of 297 amino acids and it appears to prevent mutations and other injuries to DNA via the nucleotide excision and repair pathway. This process involves removing the modified nucleotide together with several adjacent nucleotides from the damaged strand (excision), and this is followed by filling in the resulting gap through the activity of DNA polymerase (repair synthesis). The ERCC1 protein is considered to be part of a functional complex with the ERCC4, ERCC11 and XPF proteins, and this complex may be required for both nucleotide excision-repair and recombination repair (10). The cytotoxicity of cisplatin has been associated with its induction of DNA intrastrand, interstrand and DNA-protein cross-linking.

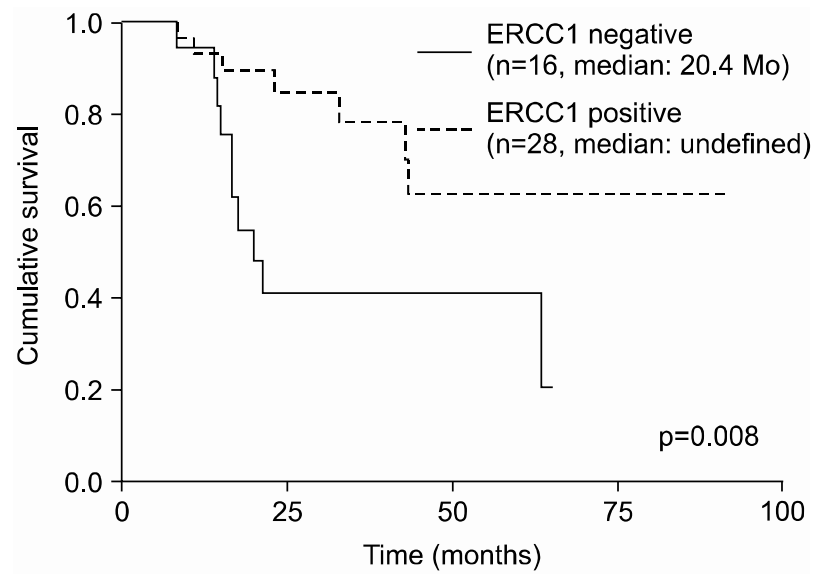

Fig. 3. Overall survival according to more and less than a $10 \%$ ERCC1 expression level as the cutoff level.

Because the ERCC1 gene product participates in the repair of the intrastrand cross-linking caused by cisplatin, human ERCC1 has a role for the gene-specific DNA repair of the cisplatininduced lesions.

Metzger et al (10). have found a significant association between the ERCC1 levels and survival after cisplatin/5-FU therapy for the patients suffering with gastric cancer. Other studies have also reported associations between higher ERCC1 expressions and worse clinical outcomes of cisplatin-based therapy for non-small cell lung cancer, esophageal cancer and ovarian cancer $(11 \sim 13)$ and for the oxaliplatin/5-FU treated colorectal cancer (14). Those studies found an association between the lower ERCC1 mRNA expression levels and the patients' improved survival after treatment.

This study investigated whether or not the ERCC1 gene overexpression is associated with the survival of the gastric cancer patients who had received cisplatin-based adjuvant chemotherapy after a curative resection. In contrast to a prior study, the DFS and OS of this study were higher in those patients showing ERCC1 gene overexpression. The causes for this were thought to be as follows.

First, the efficacy of adjuvant chemotherapy still remains a subject of some controversy. Moreover, cisplatin is effective for treating advanced gastric cancer, as seen by the response rate and survival rate, but not as an adjuvant regimen. Because of the inefficacy of cisplatin-based adjuvant chemotherapy for treating gastric cancer, the ERCC1 expression might not have a defined role as a predictive marker.

Second, the ERCC1 expression may play a role in the progression of cancer. Simon et al (16). evaluated the effects of ERCC1 expression on the overall survival for 49 patients with stage IA to IIIB non-small cell lung cancer (NSCLC) and who also underwent surgical resection. Forty-three patients received no adjuvant therapy. Their study showed that patients having a high ERCC1 expression had a better survival than did the patients having a lower ERCC1 expression. Based on their study, an efficient DNA repair mechanism affected the progression of the cancer itself. Since patients with a low ERCC1 expression had a poorer prognosis, but they responded better to chemotherapy, they were likely to benefit from chemothe- 
rapy. Further prospective studies on the ERCC1 gene expression as a prognostic marker for the DNA repair activity are needed.

Third, it is thought that there are different sensitivities and specificities between the methods used to detect the ERCC1 expression. There is growing evidence that the colorectal cancers with microsatellite instability (MSI) have a better prognosis and so they might respond differently to adjuvant chemotherapy. Up to now, the PCR-based MSI assay has been considered as the gold standard for MSI testing. Chapusot et al (17). have recently evaluated if IHC really offered an accurate, fast and cost-effective alternative to MSI testing; however, it cannot replace PCR. In prior studies, reverse transcriptase-polymerase chain reaction (RT-PCR) was used with PCR-amplified $\beta$-actin serving as an internal standard to control for the amount of RNA isolated from tumor biopsies so as to determine the relative ERCC1 levels. It was feasible to obtain the ERCC1 mRNA levels from the cDNA of 38 $(58 \%)$ primary gastric tumor specimens $(10)$. However, in the current study, 28 patients showed a positive expression of the ERCC1 gene (64.6\%) and the positive rate in our study was higher than that in the previous study. The discrepancy between using RT-PCR and IHC for detecting the ERCC1 gene may have been a confounding factor. Further prospective studies showing the association between RT-PCR and IHC are needed. Fourth, the number of metastatic lymph nodes was significantly different between the ERCC1-positive group and the ERCC1negative-group, though the disease stage of both groups was not statistically different. The number of metastatic lymph node was 13.2 in the ERCC1-negative group and it was 8.5 in the ERCC1-positive group ( $p=0.02)$.

Fifth, factors other than the ERCC1 gene may have affected the tumors' resistance to chemotherapy. Thymidylate synthase (TS), the target enzyme of the antimetabolite 5-FU, has been shown to be an independent prognostic marker of 5-FU chemotherapy for gastrointestinal tumors $(10,14)$. Decreased pyruvate kinase M2 activity has also been linked to cisplatin resistance in the human gastric carcinoma cell lines (19). However, we didn't evaluate these other factors.

Because ERCC1 participates in the repair of intrastrand cross-linking caused by cisplatin, the gene may have a pivotal role in the mechanism of cancer cell resistance to cisplatin. However, this study showed that the DFS and OS for the gastric cancer patients who received cisplatin-based adjuvant chemotherapy after a curative resection were higher for those patients who showed overexpression of the ERCC1 gene. This result was due to a limited role for cisplatin as adjuvant chemotherapy for gastric cancer, the effect of ERCC1 expression on cancer progression, the discrepancy between the RT-PCR and IHC in detecting the ERCC1 gene, the difference of the LN number and there were probably other genetic factors that could have affected the resistance to chemotherapy. Future prospective studies using the ERCC1 gene expression as a prognostic marker for the DNA repair activity are needed.

\section{CONCLUSIONS}

The disease-free survival and overall survival of the gastric cancer patients who received cisplatin-based adjuvant chemo- therapy after curative resection were higher for those patients showing ERCC1 gene overexpression. However, future prospective studies using the ERCC1 gene expression as a prognostic marker for DNA repair activity are needed.

\section{REFERENCES}

1. Shin Hr, Won YJ, Jung KW, Park JG. 2001 Annual report of the korea central cancer registry: based on registered data from 134 hospitals. Cancer Res Treat. 2004;36:19-30.

2. Richard P, Lawrence RC, William JH, Lawrence D. Wagman gastric cancer. Cancer management. 5th ed. NY: CMP media LLC; 2004. p. 259-72.

3. Rosenberg B, VanCamp L, Krigas T. Inhibition of cell division in Escherichia coli by electrolysis products from a platinum electrode. Nature. 1965;205:698-9.

4. Higby DJ, Wallace HJ Jr, Albert DJ, Holland JF. Diaminodichloroplatinum: a phase I study showing responses in testicular and other tumors. Cancer. 1974;33:1219-25.

5. Janunger KG, Hafstrom L, Nygren P, Glimelius B. A systematic overview of chemotherapy effects in gastric cancer. Acta Oncol. 2001;40:309-26.

6. Earle CC, Maroun JA. Adjuvant chemotherapy after curative resection for gastric cancer in non-Asian patients: revisiting a meta-analysis of randomized trials. Eur J Cancer. 1999;35: 1059-64.

7. Hermans J, Bonenkamp JJ, Boon MC, Bunt AM, Ohyama S, Sasako M, et al. Adjuvant therapy after curative resection for gastric cancer: meta-analysis of randomized trials. J Clin Oncol. 1993;11:1441-7.

8. Bramson J, Panasci LC. Effect of ERCC-1 overexpression on sensitivity of Chinese hamster ovary cells to DNA damaging agents. Cancer Res. 1993;53:3237-40.

9. Lee KB, Parker RJ, Bohr V, Cornelison T, Reed E. Cisplatin sensitivity/resistance in UV repair-deficient Chinese hamster ovary cells of complementation groups 1 and 3 . Carcinogenesis. 1993; 14:2177-80.

10. Metzger R, Leichman CG, Danenberg KD, Danenberg PV, Lenz HJ, Hayashi K, et al. ERCC1 mRNA levels complement thymidylate synthase mRNA levels in predicting response and survival for gastric cancer patients receiving combination cisplatin and fluorouracil chemotherapy. J Clin Oncol. 1998; 16:309-16.

11. Lord RV, Brabender J, Gandara D, Alberola V, Camps C, Domine $\mathrm{M}$, et al. Low ERCC1 expression correlates with prolonged survival after cisplatin plus gemcitabine chemotherapy in non-small cell lung cancer. Clin Cancer Res. 2002;8:228691.

12. Britten RA, Liu D, Tessier A, Hutchison MJ, Murray D. ERCC1 expression as a molecular marker of cisplatin resistance in human cervical tumor cells. Int J Cancer. 2000;89: 453-7.

13. Codegoni AM, Broggini M, Pitelli MR, Pantarotto M, Torri $\mathrm{V}$, Mangioni C, et. al. Expression of genes of potential importance in the response to chemotherapy and DNA repair in patients with ovarian cancer. Gynecol Oncol. 1997;65: 130-7.

14. Shirota Y, Stoehlmacher J, Brabender J, Xiong YP, Uetake H, Danenberg KD, et al. ERCC1 and thymidylate synthase mRNA levels predict survival for colorectal cancer patients receiving combination oxaliplatin and fluorouracil chemotherapy. J Clin Oncol. 2001;19:4298-304.

15. Beahrs OH, Henson DE, Hutter RV, Kennedy BJ. Manual for staging of cancer. american joint committee on cancer. 5th ed. 
Philadelphia: JB Lippincott; 1997. p. 81-7.

16. Simon GR, Sharma S, Canter A, Smith P, Bepler P. ERCC1 expression is a predictor of survival in resected patients with non-small cell lung cancer (NSCLC). Chest. 2005;127:978-83.

17. Chapusot C, Martin L, Puig PL, Ponnelle T, Cheynel N, Bouvier AM, et al. What is the best way to assess microsatellite instability status in colorectal cancer? Am J Surg Pathol. 2004;28:1553-9.
18. Gurubhagavatula S, Liu G, Park S, Zhou W, Su L, Win JC, et al. XPD and XRCC1 genetic polymorphisms are prognostic factors in advanced non-small-cell lung cancer patients treated with platinum chemotherapy. J Clin Oncol. 2004;22:2594-601.

19. Yoo BC, Ku JL, Hong SH, Shin YK, Park SY, Kim HK, et al. Decreased pyruvate kinase M2 activity linked to cisplatin resistance in human gastric carcinoma cell lines. Int $\mathrm{J}$ Cancer. 2004;108:532-9. 